\title{
Effect of Angle of Attack on Stability Derivatives of a Delta Wing with Straight Leading Edge in Supersonic Flow
}

\author{
Asha Crasta ${ }^{1,2}$ and S. A. Khan ${ }^{3}$ \\ 1. Research Scholar, Jain University, Bangalore, Karnataka, India \\ 2. Sr. Assistant Professor, Mangalore Institute of Technology and Engineering, Moodabidri, Karnataka, India. \\ 3. Principal, Z. H. College of Engineering \& Technology, AMU, Aligarh, UP, India
}

\begin{abstract}
In the Present paper effect of angle of incidence on pitching derivatives of a delta wing with Straight leading edges for attached shock case in Supersonic Flow has been studied. A Strip theory is used in which strips at different span wise location are independent. This combines with similitude to give a piston theory. From the results it is seen that with the increase in the Mach number, there is a decrement of stiffness as well as the damping derivatives in pitch for all the Mach number tested, however, the magnitude of decrement for different inertia level will differ. It is seen that with the increase in the angle of attack both stiffness and damping derivatives increase linearly, nevertheless, this linear behavior limit themselves for different Mach numbers. For Mach number $M=2$, this limiting value of validity is fifteen degrees, for Mach 2.5 \& 3, it is twenty five degrees, whereas, for Mach 3.5 \& 4 it becomes thirty five degrees. When these stability derivatives were considered at various pivot positions, namely $h=0.0,0.2,0.4,0.6,0.8$, and 1.0. After scanning the results it was observed that with the shift of the pivot position from the leading edge to the trailing edge, the magnitude of both the stability derivatives were decreasing progressively with the pivot position. Results have been obtained for supersonic flow of perfect gases over a wide range of angle of attack and Mach number. The effect of real gas, leading edge bluntness of the wing, and secondary wave reflections are neglected.
\end{abstract}

Keywords: angle of incidence, delta wing, Pitching derivatives, Supersonic Flow, Straight leading edge

\section{Introduction}

The theories for steady delta wings in supersonic/hypersonic flow with shock wave attached were given by Pike [1] and Hui [2]. Carrier [3] and Hui [4] gave exact solutions for 2-D flow in the case of an oscillating wedge and for an oscillating flat plate were given by Hui [5], which is valid uniformly for all supersonic Mach numbers and wedge angles or angles of attack with attached shock wave. Hui [5] also calculated pressure on the compression side of a flat delta.

The importance of dynamic stability at large incidence during re-entry or maneuver has been pointed out by Orlik-Ruckemann [6]. The shock attached relatively high aspect ratio delta is often preferred for its high lift to drag ratio.

Hui and Hemdan [7] have studied the unsteady shock detached case in the context of thin shock layer theory. Liu and Hui [8] have extended Hui's [5] theory to a shock attached delta wing in pitch. Light hill [9] has developed a "Piston Theory" for oscillating airfoils at high Mach numbers. A parameter $\delta$ is introduced, which is a measure of maximum inclination angle of Mach wave in the flow field. It is assumed that $\mathrm{M}_{\infty} \delta$ is less than or equal to unity (i.e. $M_{\infty} \delta \leq 1$ ) and is of the order of maximum deflection of a streamline. Light hill [9] likened the 2-D unsteady problem to that of a gas flow in a tube driven by a piston and termed it "Piston Analogy".

Ghosh [10] has developed a large incidence 2-D hypersonic similitude and piston theory. It includes Light hill's [9] and Mile's [11] piston theories. Ghosh and Mistry [12] have applied this theory of order of $\phi^{2}$ where $\phi$ is the angle between the attached shock and the plane approximating the windward surface. For a plane surface, $\phi$ is the angle between the shock and the body. The only additional restriction compared to small disturbance theory is that the Mach number downstream of the bow shock is not less than 2.5.

Ghosh [13] has obtained a similitude and two similarity parameters for shock attached oscillating delta wings at large incidence. Crasta and Khan have extended the Ghosh similitude to Hypersonic/supersonic flows past a planar wedge [14] and [18] and Non planar wedge [20], [21], and [22]. Crasta and Khan have obtained stability derivatives in pitch and roll of a delta wing with straight leading edge [23] and [24] and curved leading edges for supersonic flows [15] and Hypersonic flows [16]. Crasta and Khan have studied the effect of angle of incidence on pitching derivatives and roll of a damping derivative of a delta wing with curved leading edges for an attached shock case [17] and [27]. Further in all cases stability derivatives in Newtonian limit have been calculated by Crasta and Khan [19], [25], and [26]. In the present analysis the effect of angle of attack on the stiffness and the damping derivative for hypersonic flows has been studied and results are obtained are shown in the section to follow. Further, the pressure on the lee surface is assumed zero. 


\section{Analysis:}

A thin strip of the wing, parallel to the centerline, can be considered independent of the $\mathrm{z}$ dimension when the velocity component along the $\mathrm{z}$ direction is small. This has been discussed by Ghosh's. The strip theory combined with Ghosh's large incidence similitude leads to the "piston analogy" and pressure P on the surface can be directly related to equivalent piston mach no. Mp. In this case both $\mathrm{Mp}$ and flow deflections are permitted to be large. Hence light hill piston theoryor miles strong shock piston theory cannot be used but Ghosh's piston theory will be applicable.

$$
\frac{P}{P_{\infty}}=1+A M_{P}{ }^{2}+A M_{P}\left(B+M_{P}{ }^{2}\right)^{\frac{1}{2}} \text {, Where } P_{\infty} \text { is free stream pressure }
$$

Since strips at different span wise location are assumed independent of each other, the strip can be considered as a flat plate at an angle of attack. The angle of incidence is same as that of wing. Angle $\phi$ is the angle between the shock and the strip. A piston theory which has been used in equation(1) has been extended to supersonic flow. The expression is given below.

$$
\frac{p}{p_{\infty}}=1+A\left(\frac{M_{p}}{\cos \phi}\right)^{2}+A\left(\frac{M_{p}}{\cos \phi}\right)\left(B+\left(\frac{M_{p}}{\cos \phi}\right)^{2}\right)^{\frac{1}{2}}
$$

Where $p_{\infty}$ is free stream pressure, $A=\frac{(\gamma+1)}{4}, B=\left(4 /(\gamma+1)^{2}, \gamma\right.$ is the specific heat ratio and $M_{p}=$ the local piston Mach number normal to the wedge surface.

\section{Pitching moment derivatives:}

Let the mean incidence be $\alpha_{0}$ for the wing oscillating in pitch with small frequency and amplitude about an axis $x_{0}$. The piston velocity and hence pressure on the windward surface remains constant on a span wise strip of length $2 \mathrm{z}$ at $\mathrm{x}$. The pressure on the lee surface is assumed Zero. Therefore the nose up moment is

$m=-2 \int_{0}^{c} p \cdot z\left(x-x_{0}\right) d x$

The stiffness derivative is non-dimensionalized by dividing with the product of dynamic pressure, wing area and chord length.

$$
\therefore-C_{m_{\alpha}}=\frac{2}{\rho_{\infty} U_{\infty}^{2} C^{3} \cot \varepsilon}\left(-\frac{\partial m}{\partial \alpha}\right)_{\substack{\alpha=\alpha_{0} \\ q=0}}
$$

The damping derivative is non-dimensionalised by dividing with the product of dynamic pressure, wing area, chord length and characteristic time factor $\left(\frac{c}{U_{\infty}}\right)$

$$
\therefore-{ }^{C} m_{q}=\frac{2}{\rho_{\infty} U_{\infty} c^{4}(\cot \varepsilon)}\left(-\frac{\partial m}{\partial q}\right)_{\substack{\alpha=\alpha_{o} \\ q=0}}
$$

The local piston Mach number normal to the wing surface is given by

$$
M_{p}=M_{\infty} \sin \alpha+\frac{q}{a_{\infty}}\left(x-x_{o}\right)
$$

Where $\rho_{\infty}, a_{\infty}$ are density and velocity of sound in the free stream. Combining (2) through (6), differentiation under the integral sign is performed.

Definingx $_{0}=\mathrm{hL}, S_{1}=\frac{M_{\infty} \sin \alpha_{o}}{\cos \phi}$, the derivatives in pitch of a delta wing become equal to

$$
-C_{m_{\alpha}}=\frac{\sin \alpha_{O} \cos \alpha_{o} f\left(S_{1}\right)}{\cos ^{2} \phi}\left[\left(\frac{2}{3}-h\right)\right]
$$




$$
-C_{m_{q}}=\frac{\sin \alpha_{o} f\left(S_{1}\right)}{\cos ^{2} \phi}\left[\left(h^{2}-\frac{4}{3} h+\frac{1}{2}\right)\right]
$$

Where $f\left(S_{1}\right)=\frac{(r+1}{2 S_{1}}\left[2 S_{1}+\left(B+2 S_{1}^{2} /\left(B+2 S_{1}^{2}\right)^{\frac{1}{2}}\right]\right.$

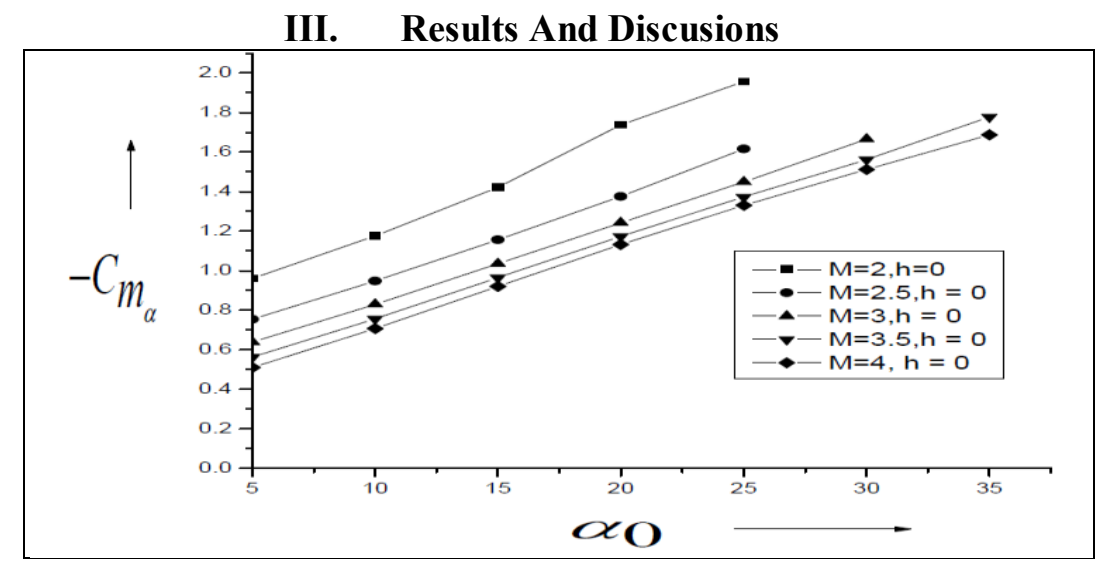

Fig. 1: Variation of Stiffness derivative with angle of attack

Fig. 1 presents the results of stiffness derivative and it's variations with angle of attack in the range from five degrees to thirty five degrees for Mach numbers in the range $\mathrm{M}=2,2.5,3,3.5$ and 4 for pivot position $\mathrm{h}=0.0$. Since the pivot position $\mathrm{h}=0.0$; which implies that this point is positioned at the leading edge itself which will result in maximum value of the stiffness derivative due to the moment arm available for the restoring moment. It is also observed that there is a sudden drop in the magnitude of the stiffness derivative, and this may be attributed due to the enhancement in the inertia level. It is also observed that for the lowest Mach number namely $\mathrm{M}=2$, the present theory may not be valid for angle of attack beyond fifteen degrees. It is found that when the Mach number was increased from $\mathrm{M}=2$ to 2.5 , resulting in the reduction in the stiffness derivative by twenty one percent. Similarly the Mach numbers were further increased from $M=2.5$ to 3,3 to 3.5 and 3.5 to 4 , they result in the decrement in the stiffness derivative by thirteen, sixteen, and ten percent, respectively. It is well know from the shock expansion theory that for a given Mach number and angle of attack two solutions exists, one is the weak solution and the other one is the strong solution. Since we considering the shock attached case hence, we will consider the weak solution only. This may be the reason for this trend for stiffness derivative at different Mach numbers.

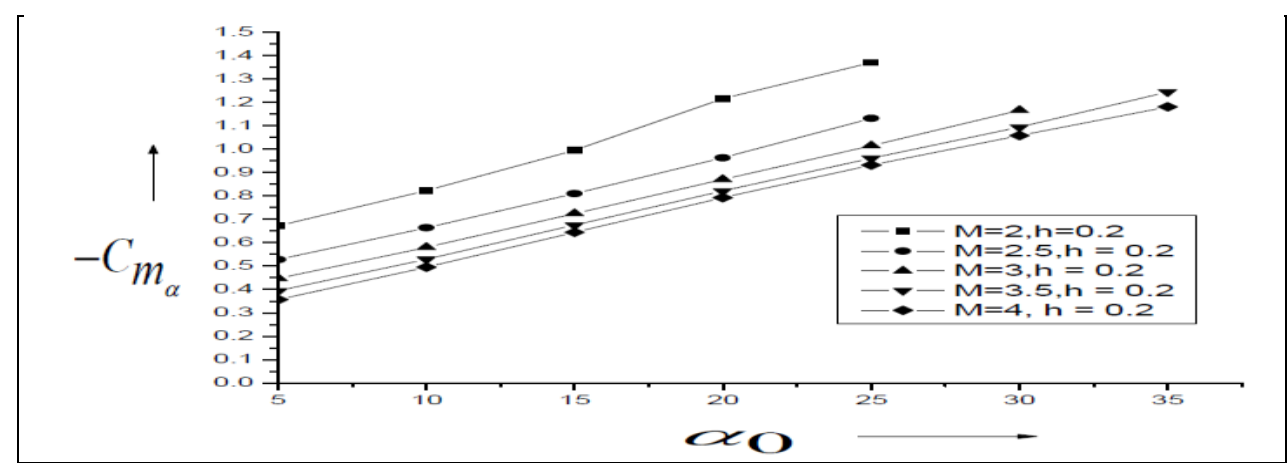

Fig. 2: Variation of Stiffness derivative with angle of attack

Fig. 2 presents the similar results as discussed earlier while discussing the figure 1. In this case the pivot position is at $\mathrm{h}=0.2$, which has shifted towards the trailing edge by twenty percent thereby reducing the moment arm keeping all other parameters same. It is seen that there is a continuous decrement in the magnitude of the stiffness derivatives in the range nineteen, fourteen, eleven, and thirteen percent for same Mach number range as discussed above. The validity of the angle of attack range too remains the same. 


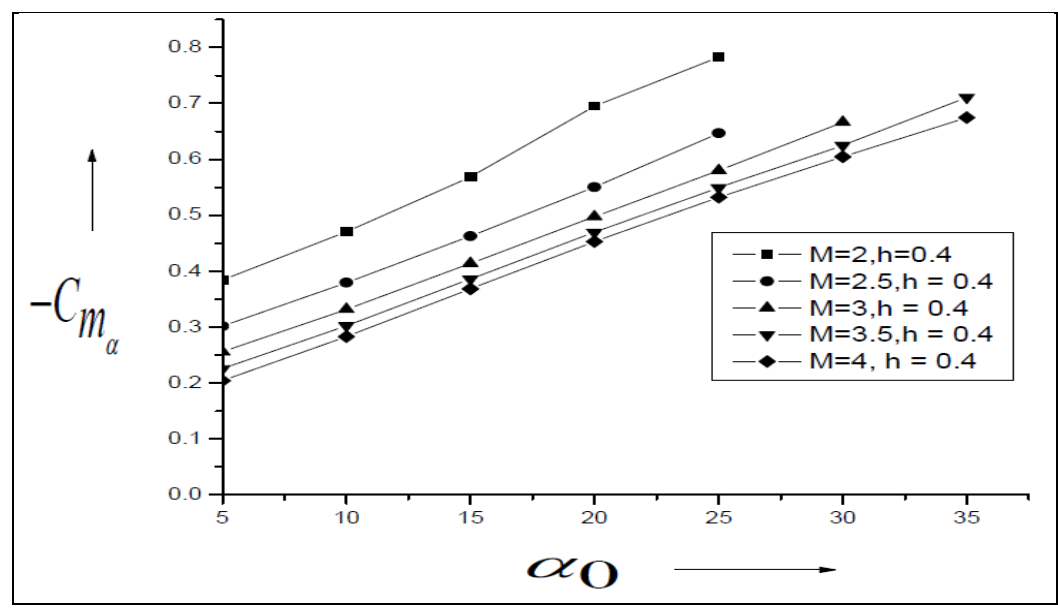

Fig. 3: variation of Stiffness derivative with angle of attack

Figure 3 presents the results for $\mathrm{h}=0.4$, for stiffness derivatives when the pivot position has been shifted further towards the trailing edge. Since all the parameters remain same, hence; the results in figure 3 reflect the effect of the location of the pivot position; and the reduction in the value of the stiffness derivatives amounts to be twenty one, seventeen, twelve, and nine percent for the same range of the Mach numbers. Also, the dependency on the angle of attack and its trend remains the same.

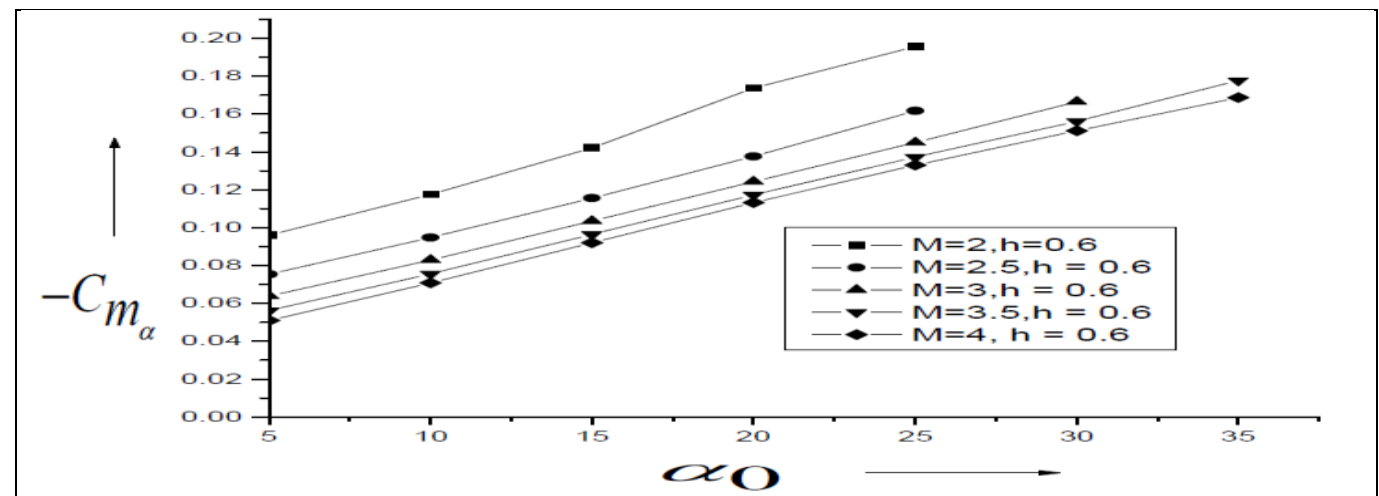

Fig. 4: Variation of Stiffness derivative with angle of attack

Fig. 4 presents the results of stiffness derivatives with angle of attack for $\mathrm{h}=0.6$. From the figure it is seen that the magnitude of the stiffness derivative has reduced significantly as this value of the pivot position is within the range of the aerodynamic/center of pressure. The decrement in the stiffness derivative are twenty six, thirteen, sixteen and nine percent, also the trend with angle of incidence are on the similar lines as discussed earlier.

Fig. 5 presents the results of stiffness derivatives with angle of attack for $h=0.8$. From the figure it is seen that the magnitude of the stiffness derivative has further reduced as this value of the pivot position is behind the aerodynamic/center of pressure resulting in very small value of the moment arm and hence the small value of the stiffness derivative. The decrements in the stiffness derivative for other Mach numbers are seventeen, seventeen, twelve and nine percent, also the trend with angle of incidence are on the similar lines as discussed earlier. 


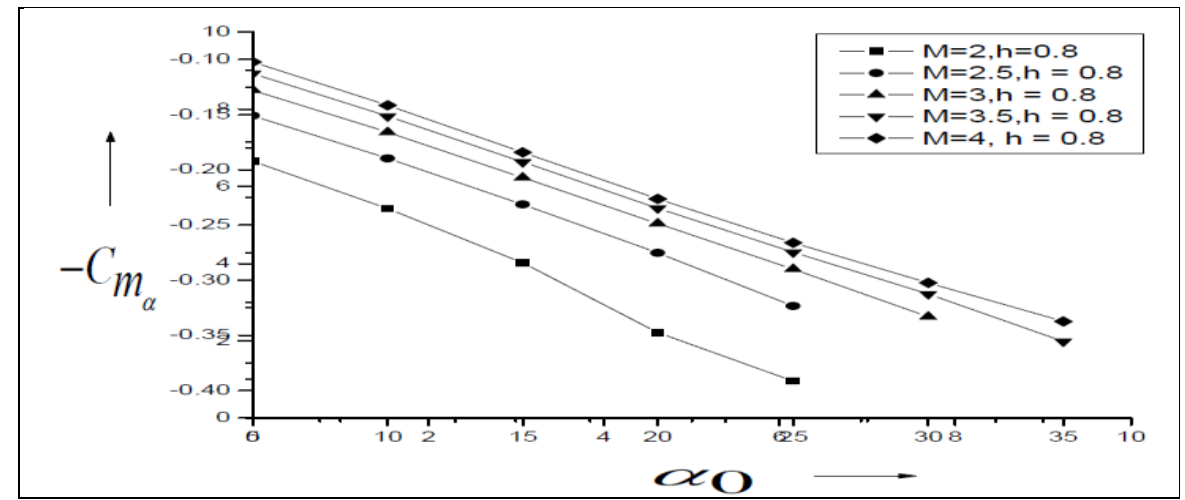

Fig. 5: Variation of Stiffness derivative with angle of attack

Fig. 6 presents the results of stiffness derivatives with angle of attack for $h=1.0$. From the figure it is seen that the magnitude of the stiffness derivative has reduced significantly as this value of the pivot position is far away from the aerodynamic/center of pressure and positioned exactly at the trailing edge. The decrements in the stiffness derivative are nineteen, fifteen, and twenty six and twenty three percent, also the trend with angle of incidence are on the similar lines.

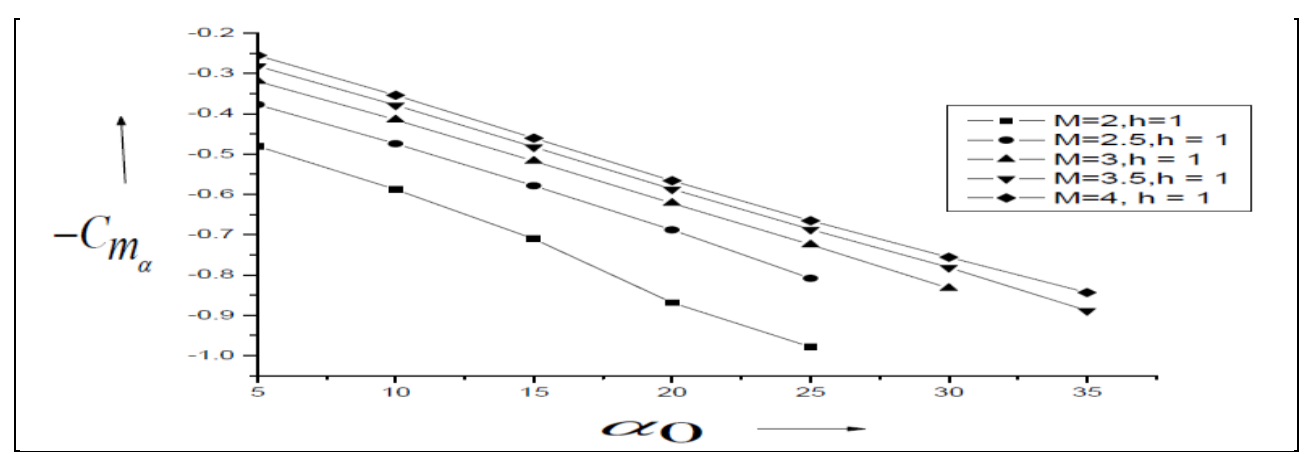

Fig.6: Variation of Stiffness derivative with angle of attack

Fig. 7 presents the results of damping derivatives in pitch and it's variations with angle of attack in the range from five degrees to thirty five degrees for Mach numbers in the range $M=2,2.5,3,3.5$ and 4 for pivot position $h=0.0$. Since the pivot position is $h=0.0$; which means that this point is positioned at the leading edge of the wing which will result in maximum value of the damping derivative due to the maximum value of the moment arm available for the restoring moment. It is also observed that there is a huge reduction in the magnitude of the damping derivative, and this may be attributed due to the increment in the inertia level. The percentage decrease in damping derivatives are twenty two, sixteen, eight, and eleven percent, respectively. It is also observed that for the lowest Mach number namely $\mathrm{M}=2$, it seems that the present theory is not valid for angle of attack beyond fifteen degrees. The trend for other angles of attack remains the same as discussed earlier.

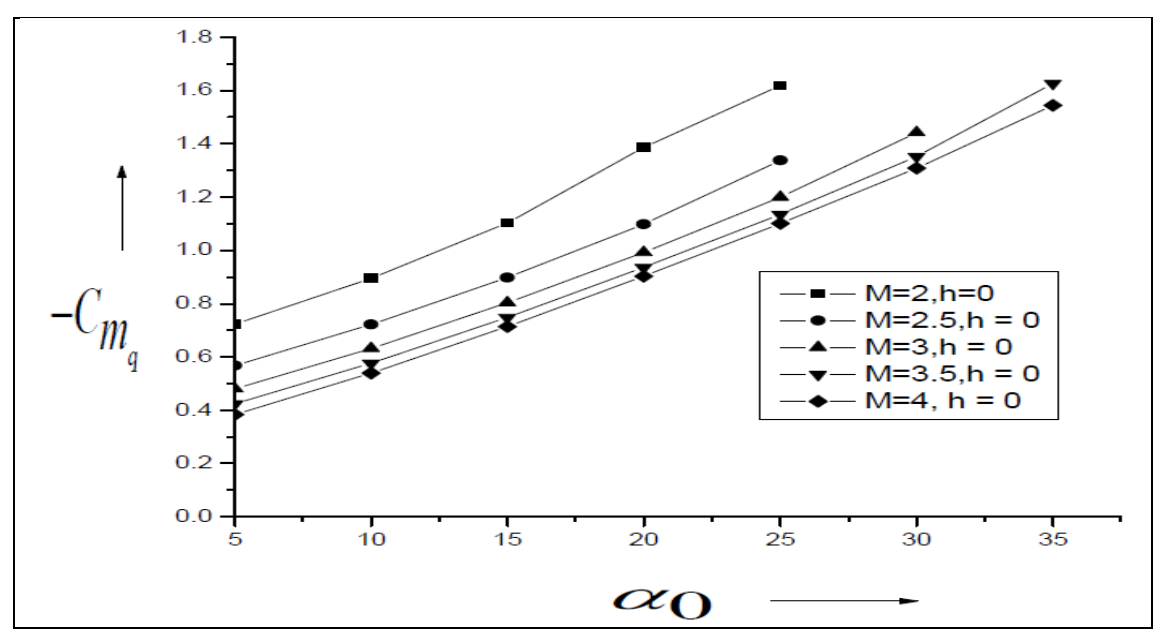

Fig. 7: Variation of damping derivative with angle of attack 
Fig. 8 presents the similar results as discussed earlier while discussing the figure 7 . In this case the pivot position is at $\mathrm{h}=0.2$, which has shifted towards the trailing edge by twenty percent thereby reducing the moment arm keeping all other parameters same. It is seen that there is a continuous decrement in the magnitude of the damping derivatives in the range twenty three, sixteen, twelve, and nine percent for same set of parameters as discussed above. The validity of the angle of attack range too remains the same.

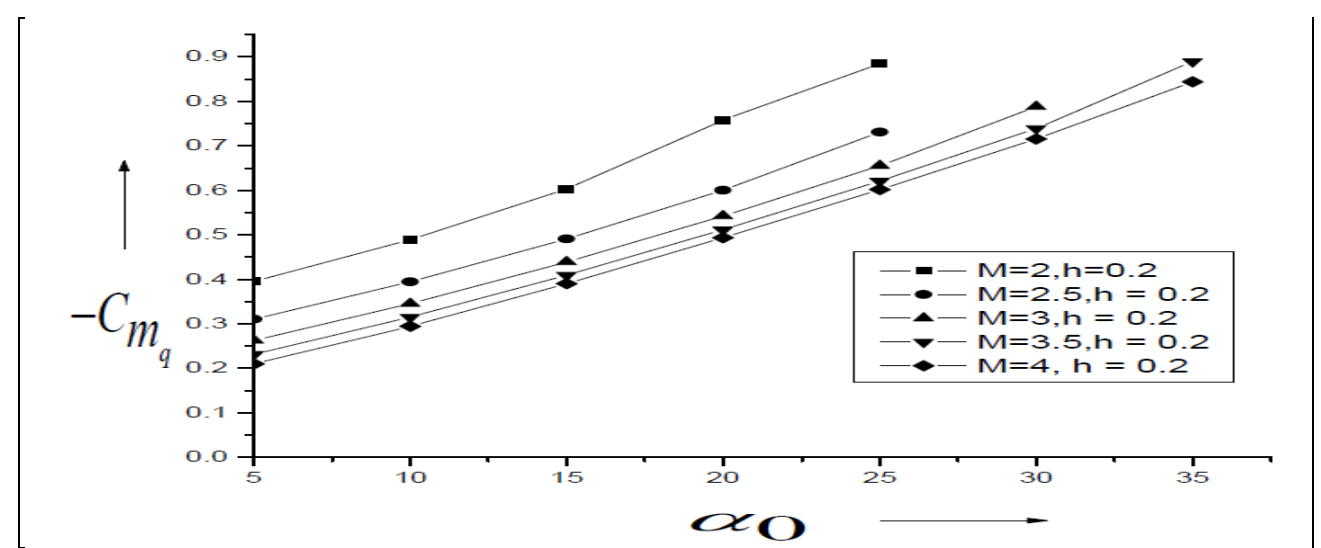

Fig. 8: Variation of damping derivative with angle of attack

Figure 9 presents the results for $\mathrm{h}=0.4$, for damping derivatives in pitch when the pivot position has been shifted further shifted towards the trailing edge. Since all the parameters are kept same, hence; the results in figure 8 reflect the effect of the location of the pivot position in the values of the damping derivatives; and the reduction in the value of the damping derivatives in pitch amounts to be nineteen, seventeen, eight, and eighteen percent for the same range of the Mach numbers as well as the angles of attack. It is also seen that the dependency on the angle of attack and its trend remains on the similar lines as seen earlier.

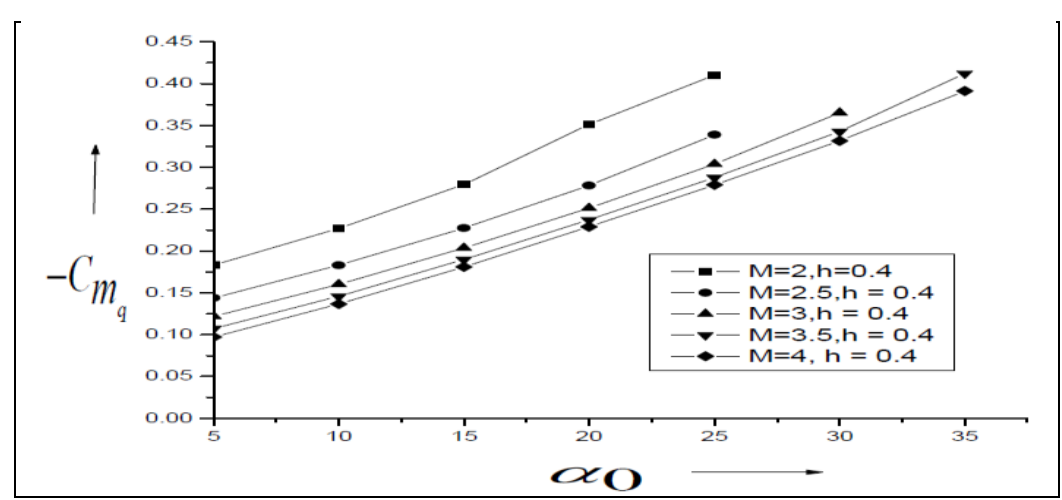

Fig. 9: Variation of damping derivative with angle of attack

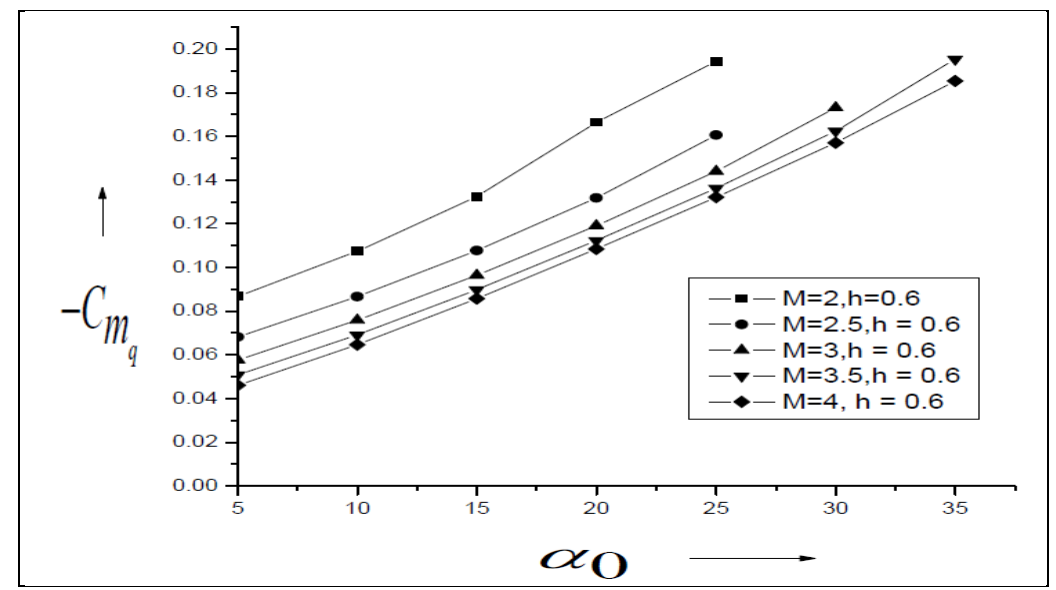

Fig. 10: Variation of damping derivative with angle of attack 
Fig. 10 presents the results of damping derivatives in pitch with angle of attack for $h=0.6$. From the figure it is seen that the magnitude of the damping derivative has reduced significantly as this value of the pivot position is very close to the aerodynamic/center of pressure. The decrements in the stiffness derivative are twenty, fourteen, seventeen and ten percent, also the trend with angle of incidence are on the similar lines as discussed earlier.

Fig. 11 presents the results of damping derivatives with angle of attack for $h=0.8$. From the figure it is seen that the magnitude of the stiffness derivative has further reduced as this value of the pivot position is behind the aerodynamic/center of pressure resulting in very small value of the moment arm and hence the small value of the stiffness derivative. The decrements in the damping derivatives are twenty three, seven, twenty five, and seven percent, also the variations with angle of incidence are on the similar lines.

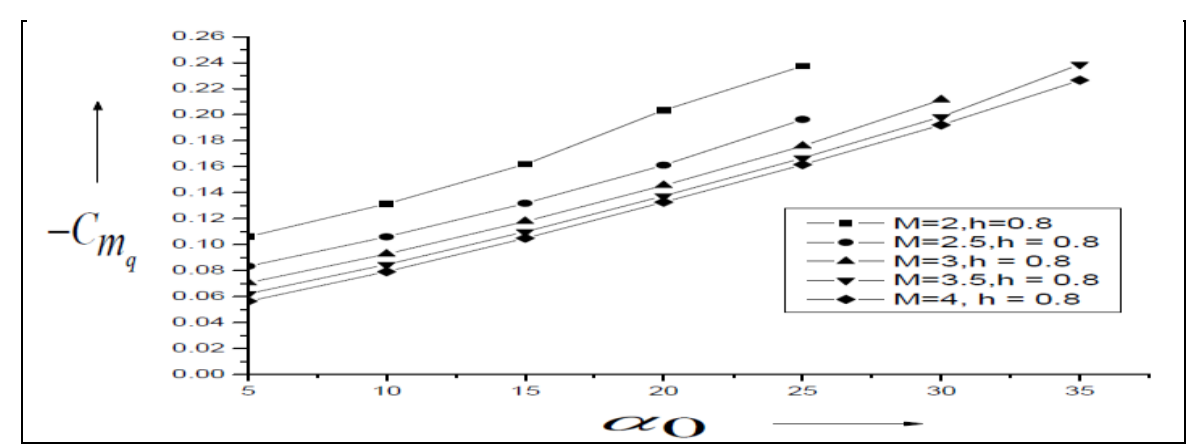

Fig. 11: Variation of damping derivative with angle of attack

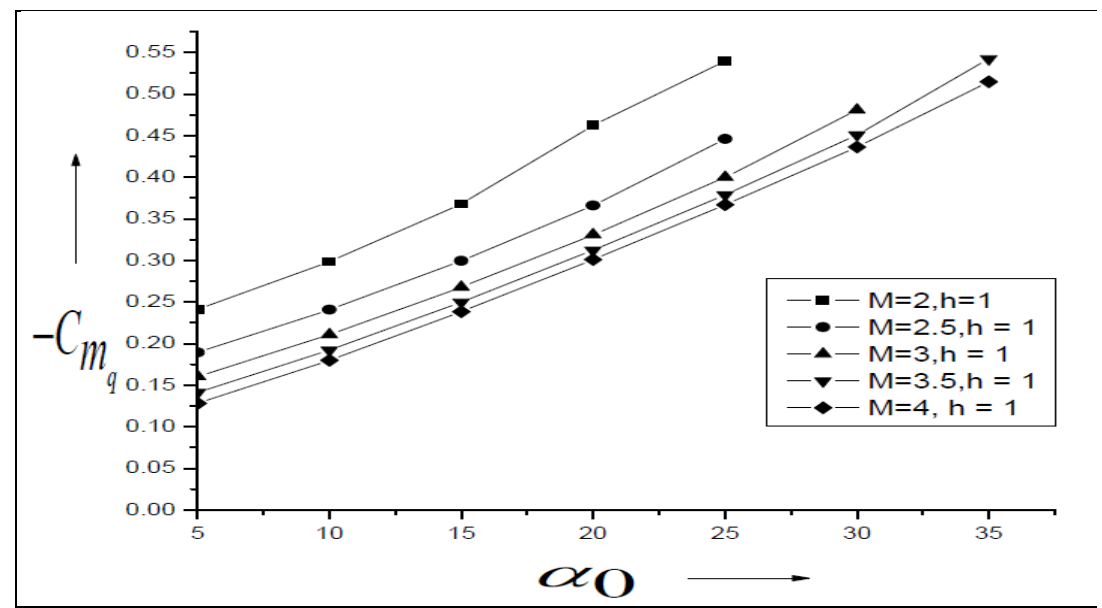

Fig. 12: Variation of damping derivative with angle of attack

Fig. 12 presents the results of damping derivatives with angle of attack for $h=1.0$. From the figure it is seen that the magnitude of the damping derivative has reduced significantly as this value of the pivot position is far away from the aerodynamic/center of pressure and positioned exactly at the trailing edge of the wing. The decrements in the stiffness derivative are twenty one, thirteen, fifteen, and twenty three percent, also the trend with angle of incidence are on the similar lines.

\section{Conclusions}

From the above results we can draw the following conclusions:

- From the results it is seen that with the increase in the Mach number, it results in the decrement of stiffness as well as the damping derivatives in pitch for all the Mach number tested, however, the magnitude of decrement for different inertia level will differ.

- It is seen that with the increase in the angle of attack both stiffness and damping derivatives increase linearly, nevertheless, this linear behavior limit themselves for different Mach numbers. For Mach number $\mathrm{M}=2$, this limiting value of validity is fifteen degrees, for Mach $2.5 \& 3$, it is twenty five degrees, whereas, for Mach $3.5 \& 4$ it becomes thirty five degrees. This restriction was due to the limitation of the present theory which is valid as long as the shock wave is attached with the wing leading edges.

- When these stability derivatives were considered at various pivot positions, namely $\mathrm{h}=0.0,0.2,0.4,0.6$, 0.8 , and 1.0. After scanning the results it was observed that with the shift of the pivot position from the 
leading edge to the trailing edge, the magnitude of both the stability derivatives were decreasing progressively with the pivot position.

- Second order shock expansion theory gives two solutions for any given Mach number and angle of attack. In view of the restrictions imposed by the present theory we should consider only weak solution and in no case we can take the strong solutions.

- The effect of real gas, leading edge bluntness of the wing, and secondary wave reflections are neglected.

\section{References}

[1] Pike. J, "The pressure on flat and anhydral delta wings with attached shock waves", The Aeronautical Quarterly, November 1972, XXIII, Part 4, pp. 253-262.

[2] Hui, W.H., "Stability of Oscillating Wedges and Caret Wings in Hypersonic and Supersonic Flows", AIAA Journal, Vol. 7, Aug. 1969, pp.1524-1530.

[3] Carrier, G.F. 1949, “The oscillating Wedge in Supersonic stream”, Journal of Aeronautical Sciences, Vol. 16, No. 3, pp. 150-152, March.

[4] Hui, W. H., "Supersonic/hypersonic flow past on oscillating flat plate at high angles of attack", ZAMP, Vol. 29, 1978, pp. 414-427.

[5] Hui, W. H., "Supersonic and hypersonic flow with attached shock waves over delta wings", Proc of Royal Society, London, 1971, A. 325 , pp. $251-268$.

[6] Orlik-Ruckemann, K. J., "Dynamic stability testing of aircraft needs versus capabilities", Progress in the Aerospace Sciences, Academic press, N.Y., 1975, 16, pp. 431-447.

[7] Hui, W. H. and Hemdan, H. T., "Unsteady hypersonic flow over delta wings with detached shock waves", AIAA Journal, April 1976,14 , pp. 505-511.

[8] Lui, D. D. and Hui W. H., "Oscillating delta wings with attached shock waves,” AIAA Journal”, June 1977, 15, 6, pp. 804-812.

[9] Light Hill, M. J., "Oscillating Aerofoil at High Mach Numbers", Journal of Aeronautical Sciences, Vol. 20, June 1953, pp. 402-406.

[10] Ghosh K, "A new similitude for aerofoil in hypersonic flow", Proc of the 6th Canadian congress of applied mechanics", Vancouver, $29^{\text {th }}$ May $3^{\text {rd }}$ June, 1977 , pp. 685-686.

[11] Miles, J. W., "Unsteady flow at hypersonic speeds, Hypersonic flow”, Butter worths Scientific Publications, London, 1960, pp. 185197.

[12] Ghosh, K and Mistry B. K., "Large incidence hypersonic similitude and oscillating non-planar wedges", AIAA Journal, August $1980,18,8$, pp. 1004-1006.

[13] Ghosh K., "Hypersonic large deflection similitude for oscillating delta wings", The Aeronautical journal, October, 1984, pp. 357361 .

[14] Asha Crasta and Khan S. A., "High Incidence Supersonic similitude for Planar wedge", International Journal of Engineering research and Applications, Vol. 2, Issue 5, September-October 2012, pp. 468-471.

[15] Khan S. A. and Asha Crasta, "Oscillating Supersonic delta wings with curved leading edges", Advanced Studies in Contemporary mathematics, Vol. 20 (2010), No.3, pp. 359-372.

[16] Asha Crasta and S. A. Khan, "Estimation of Stability derivatives of an Oscillating Hypersonic delta wings with curved leading edges",Vol.3, Issue 3, December 2012, pp. 483-492.,

[17] Asha Crasta and S. A. Khan, "Effect of Angle of Incidence on Stability derivatives of a wing", International Journal for Research in Applied Science and Engineering Technology, Vol. 2, Issue V, May 2014, pp. 411-422.

[18] Asha Crasta and S. A. Khan, "Hypersonic Similitude for Planar Wedges", International Journal of Advanced Research in Engineering and Technology, Volume 5, Issue2, February 2014, pp. 16-31.

[19] Asha Crasta and S. A. Khan, "Estimation of Stability Derivatives for a Planar Wedge in the Newtonian Limit", IOSR Journal of Mathematics, Volume 10, Issue 2, Version I, March-April 2014, pp. 01-06.

[20] Asha Crasta and S. A. Khan, "Effect of Mach number on Stiffness and Damping Derivatives for Oscillating Hypersonic Non-Planar Wedge", IOSR Journal of Mechanical and Civil Engineering, Volume 11, Issue 2, Ver. VIII, March- April 2014, pp. 04-11.

[21] Asha Crasta and S. A. Khan,"Effect of Angle of Incidence on Stiffness and Damping derivatives for Oscillating Hypersonic Nonplanar Wedge", International Journal for Research in Applied Science and Engineering Technology, Vol. 2, Issue IV, April 2014, pp. 229-242.

[22] Asha Crasta and S. A. Khan, "Supersonic Similitude for Oscillating Non-Planar wedge", IOSR Journal of Mathematics, Volume 10, Issue 2, Ver.VI, March-April 2014, pp.15-24.

[23] Asha Crasta, M. A. Baig, S. A. Khan , 'Estimation of Stability derivatives of a Delta wing in Hypersonic flow', International Journal of Emerging trends in Engineering and Developments, Vol. 6, Issue 2, September 2012, pp. 505-516.

[24] Asha Crasta and S. A. Khan, "Oscillating Supersonic delta wing with Straight Leading Edges", International Journal of Computational Engineering Research, Vol. 2, Issue 5, September 2012, pp. 1226-1233.

[25] Asha Crasta and S. A. Khan, 'Stability derivatives of a Delta Wing with Straight leading edge in the Newtonian Limit', International Journal of Engineering research and Applications, Vol. 4, Issue 3, Version 2, March 2014, pp. 32-38.

[26] Asha Crasta and S. A. Khan, "Stability Derivatives in the Newtonian Limit", International Journal of Advanced Research in Engineering and Technology, Volume 4, Issue 7, November-December 2013, pp. 276-289.

[27] Asha Crasta, S. A. Khan , 'Effect of angle of incidence on Roll damping derivative of a Delta Wing', International Journal of Emerging trends in Engineering and Developments Volume 2, Issue 4, March 2014, pp. 343-356.

[28] Asha Crasta, S.A.Khan, 'Effect of sweep angle on Roll damping derivative of a Delta Wing', International Journal of Emerging trends in Engineering and Developments Volume 5, Issue 4, August-September 2014, pp. 45-55. 